\title{
Attentions du clergé bas-canadien aux personnes nécessiteuses, 1800-1840
}

\section{Lucien Lemieux}

Volume 50, numéro 1, 1983

Bilan de l'histoire religieuse au Canada

Canadian Catholic History: A survey

URI : https://id.erudit.org/iderudit/1007041ar

DOI : https://doi.org/10.7202/1007041ar

Aller au sommaire du numéro

Éditeur(s)

Les Éditions Historia Ecclesiæ Catholicæ Canadensis Inc.

ISSN

0318-6172 (imprimé)

1927-7067 (numérique)

Découvrir la revue

Citer cet article

Lemieux, L. (1983). Attentions du clergé bas-canadien aux personnes

nécessiteuses, 1800-1840. Sessions d'étude - Société canadienne d'histoire de

l'Église catholique, 50(1), 159-175. https://doi.org/10.7202/1007041ar

Tous droits réservés @ Les Éditions Historia Ecclesiæ Catholicæ Canadensis Inc., 1983
Ce document est protégé par la loi sur le droit d'auteur. L'utilisation des services d'Érudit (y compris la reproduction) est assujettie à sa politique d'utilisation que vous pouvez consulter en ligne.

https://apropos.erudit.org/fr/usagers/politique-dutilisation/ 


\section{Attentions du clergé bas-canadien aux personnes nécessiteuses, $1800-1840$}

\section{INTRODUCTION}

Confiner les activités journalières des curés aux célébrations liturgiques et sacramentelles, ainsi qu'à leurs exercices spirituels individuels, relève du mythe ou de l'ignorance. À toutes les époques, les prêtres de paroisse ont exercé leur ministère selon les quatre axes fondamentaux de la paroisse: liturgie et sacrements, éducation de la foi des adultes et des jeunes, fraternité et vie communautaire, promotion humaine.

Cette dernière dimension rejoint toutes les facettes de la vie en société. Elle est le lieu d'engagement des membres de l'Église dans la construction du monde. Elle se situe d'une certaine manière aux confins de ce qui leur est propre et de ce qui relève de la société. Étant donné les nombreuses avenues de suppléance qui sillonnaient le Bas-Canada au début du $19^{\mathrm{e}}$ siècle, le clergé se sentait responsable de plusieurs domaines de la vie sociale, en particulier celui du système scolaire. Les options politiques des citoyens ne lui étaient pas étrangères, ni le courant nationaliste qui traversait la population francophone. Ces divers aspects de la pastorale sociale ont été traités de multiples façons par différents auteurs.

Les propos suivants se limiteront donc à refléter la pastorale de la promotion humaine sous son angle caritatif. L'attention aux démunis a été de tout temps considérée comme profondément évangélique. Le curé François-Xavier Côté n'était pas le seul à écrire ainsi à son évêque: «Du matin au soir, je suis occupé. À peine puis-je trouver le temps de dire mon bréviaire... Les uns viennent me trouver pour des dettes qu'ils doivent, les autres pour de l'assistance, etc...»' . «Les pauvres me dé-

${ }^{1} \mathrm{AAQ}$ (Archives de l'archevêché de Québec), $61 \mathrm{CD}$, Les Éboulements, 1, 5 mars 1817, à Joseph-Octave Plessis, évêque de Québec. 
vorent" ${ }^{2}$, affirme le curé de Rivière-Ouelle dix ans plus tard. Sans généraliser à partir de ces témoignages glanés parmi d'autres, on saisira davantage leur pertinence, après que l'on aura passé en revue différentes facettes de la pastorale caritative de cette époque. Celle-ci laisse place à la collaboration des laïcs, ce que les clercs auraient accepté plus difficilement dans les autres dimensions de la pastorale.

\section{FACE AUX ÉPREUVES NATURELL.FS}

\section{Les cataclysmes}

Fréquentes étaient les bénédictions de maisons ou d'autres constructions. Certaines servaient d'actions de grâce au Seigneur, d'autres de prévention contre toutes sortes de dangers possibles. Ceux-ci n'étaient cependant pas tous évités pour autant.

Le feu ravageait souvent des immeubles. Le sinistre de 1803 à Montréal rasa le collège Saint-Raphaël, l'ancienne église des Jésuites, la chapelle de Bonsecours, la vieille geôle française et toutes les maisons environnantes. "Il faut pourtant bénir la Providence, qui a subitement apaisé le vent», proclama le supérieur des Sulpiciens. «Sans cela, on ne sait jusqu'où se seraient étendus les ravages" ${ }^{3}$. D'autres incendies sérieux survinrent durant le même été à Montréal; $\mathrm{M}^{\mathrm{gr}}$ Plessis accorda la permission de prières publiques. "Si les fidèles épouvantés de ces calamités successives se tournent du côté de Dieu, c'est une occasion précieuse de les rappeler à leur religion et de les faire rentrer en euxmêmes» ${ }^{4}$; cela ranimera leur courage abattu.

Les interventions pastorales ne se limitèrent pas au domaine spirituel. Des voisins éteignant le feu et des bienfaiteurs manifestant leur générosité envers les personnes éprouvées avaient été chrétiennement éduqués dans ce sens. Comme les pertes n'étaient habituellement pas remboursées par une compagnie d'assurances, faute de cotisation de la part des gens, et qu'elles l'étaient partiellement en cas contraire, il s'agissait ordinairement d'aide à des familles démunies.

${ }^{2}$ ACAM (Archives de la chancellerie de l'archevêché de Montréal), 295.099, 827-3, 15 nov. 1827, Pierre Viau à Thomas Pépin, curé de Saint-Pierre-les-Becquets.

${ }^{3}$ ACAM, 901.114, 803-1, 13 juin 1803, Jean-Henry-Auguste Roux à Plessis.

${ }^{4} \mathrm{AAQ}, R L$ ( Registre des lettres), 3, 242, 8 août 1803, à Roux. 
Dès 1817, un lecteur du Spectateur canadien regrettait que les édifices publics ne fussent pas protégés contre les effets de la foudre grâce au "conducteur métallique" 5 de Franklin. Les églises, surtout à cause de leurs hauts clochers, préserveraient ainsi les maisons environnantes, tout en étant elles-mêmes protégées contre les éclairs et le tonnerre. Il fallut attendre le 1er septembre 1836 avant qu'un paratonnerre ne fût posé sur une église, en l'occurence celle de Sainte-Élisabeth. Les plans en furent conçus par Amable Duchaîne, un ingénieur devenu ecclésiastique. La vigilance des fabriques se concrétisait, mais l'apport financier des paroissiens ainsi que leur main-d'oeuvre bénévole demeurèrent les principaux moyens de relever tout bâtiment ou édifice ravagé par le feu.

Face aux cataclysmes, la réaction du clergé comme celle des concernés était toujours marquée par une référence à Dieu. Seul ce dernier semblait pouvoir aider les personnes qui subissaient les conséquences néfastes de tout fléau naturel. Qu'il s'agisse du besoin de la pluie causé par la sécheresse, de la diminution d'un trop grand nombre de rats ou de mulots, de la disparition de petits vers jaunes, qui mangeaient le blé dans l'épi, ainsi que des chenilles, des insectes et des sauterelles (en particulier en 1804, 1805, 1827, 1839), toute précaution humaine était accompagnée d'une supplication au Seigneur.

L'évêque se réservait le droit d'autoriser les célébrations appropriées à de telles circonstances. Il ne permettait aucune prière publique, sans vérifier les conditions suivantes: un fléau réel; une portion de gens affectés et non pas seulement quelques particuliers; des personness qui se rendent dignes des bienfaits du Seigneur en s'approchant du tribunal de la pénitence; une journée de jeûne combinée à celle de la célébration; une cérémonie «précédée d'une instruction dans laquelle les gens seront avertis de ne pas se scandaliser, s'il plaît à Dieu de ne pas les exaucer, mais de se soumettre aux ordres de la Providence, persuadés que Dieu les afflige ou pour éprouver leur fidélité, ou pour les châtier de leurs péchés, ou pour leur apprendre par des afflictions temporelles à mépriser la vie terrestre pour les jouissances de l'autre vie, où le bonheur n'est troublé par aucune contrariété» ${ }^{6}$.

\footnotetext{
${ }^{5}$ Spectateur canadien, 1 nov. 1817.

${ }^{6} \mathrm{AAQ}, R L, 9,166,4$ juin 1817 , Plessis à Joseph-Norbert Provencher, curé à Kamouraska.
} 
Souvent la vie morale et la piété des gens s'amélioraient à l'occasion et à la suite de tels malheurs, par exemple à Sainte-Scholastique en 1839. À Saint-Pierre de l'île d'Orléans, un témoignage, certifié par huit personnes, rapporte que le lendemain de la procession du 18 août 1833 , les vers étaient trouvés morts dans les champs. Les effets des prières n'apparaissaient habituellement pas de façon aussi flagrante. Il fallait plutôt fiare face à la disette et à la misère, conséquences régulières des cataclysmes naturels.

Les capitaines et les notables faisaient alors leur part, en demandant de l'aide extérieure, au moins en vue de l'ensemencement de l'année suivante. Quand le gouvernement vérifiait les besoins de chaque paroisse, il se fiait habituellement au diagnostic du curé. Beaucoup de personnes prenaient donc leurs responsabilités en faveur des déshérités, souvent à cause de leur foi chrétienne et sous l'influence des membres du clergé.

\section{Les épidémies}

Le soin pastoral des malades faisait partie intégrante de la journée des prêtres. Outre les visites requises par le sacrement d'extrêmeonction, ils se rendaient régulièrement auprès des malades pour les encourager dans leurs épreuves, les confesser de leurs fautes et les faire communier à l'Eucharistie. Les épidémies accroissaient la tâche des prêtres. La fièvre, qui débuta à l'automne 1809 à Saint-Denis, se répandit durant l'hive: dans neuf paroisses environnantes. Des victimes en moururent jusqu'au printemps suivant. L'évêque, informé par un curé dès le 22 novembre, en prévint Sommerville, l'inspecteur des hôpitaux du Bas-Canada. Les personnes âgées succombèrent plus facilement que les jeunes. Alors que les médecins essayaient de remédier à la maladie soit par des saignées, soit par le vomi, les habitants alarmés se référaient au Seigneur par le sacrement du pardon et faisaient chanter des messes. Une autre épidémie, celle de la fièvre rouge, entraîna la mort de trente-quatre personnes en janvier et février 1821 à Rivière-Ouelle, mais elle semble s'être arrêtée là.

Le 31 octobre 1831, le gouverneur Aylmer demanda aux curés: de conseiller à leurs paroissiens de ne fréquenter aucun vaisseau venant d'outre-mer et de prendre, avec les personnes influentes de leurs endroits, des mesures pour empêcher ceux qui sont à bord de tels vaisseaux de descendre au rivage avant que ceux-ci n'aient été visités par l'officier de santé. ?

${ }^{7}$ La Minerve. 
Après s'être entendu avec $\mathrm{M}^{\mathrm{gr}}$ Bernard-Claude Panet, le gouverneur décida plus tard que le vendredi 4 mai serait

\begin{abstract}
un jour de jeûne et d'humiliations publics... afin d'obtenir le pardon de nos péchés et d'offrir de la manière la plus dévote et la plus solennelle nos prières et nos supplications à la Majesté divine pour détourner les jugements rigoureux que nos provocations nombreuses nous ont mérités, et particulièrement pour implorer Dieu de détourner de nous cette grave maladie dont plusieurs parties de l'Europe sont maintenant affligées. ${ }^{8}$
\end{abstract}

Un mandement de l'évêque précisa qu'une messe solennelle serait célébrée ce jour-là dans toute église paroissiale ayant un curé résident et qu'à tous les dimanches suivants, jusqu'à nouvel ordre, des prières spéciales seraient récitées lors des messes et des saluts du Saint-Sacrement. Mais l'inévitable se produisit en 1832. Le Carrick, venu de Dublin avec un contingent d'immigrants, contamina d'abord Montréal au début de juin. Dès le 15, on avait relevé 1,200 cas, dont 230 décès, et le 26 , on comptait déjà 3,384 cas, dont 947 décès. À la mi-juillet, 1,500 citoyens de la seule ville de Québec avaient trépassé. En septembre le choléra cessa ses ravages; 7,000 personnes étaient mortes.

Les prêtres, appelés auprès des malades jours et nuits, connurent enfin du répit. Plusieurs se soucièrent, tel le curé de Québec, CharlesFrançois Baillargeon, des familles réduites à la misère. Beaucoup d'orphelins furent placés dans de bonnes familles. Des organismes bénévoles, telles les Dames de la charité, s'occupèrent aussi des veuves et des orphelins.

Le même fléau rebondit deux ans plus tard, comme il le referait en 1849,1851 et 1854 . Les malades s'avérèrent moins nombreux, mais la mort les frappait presque tous. "C'est une vraie peste», affirme un curé; "les uns l'attrapent par fréquentation avec les malades ou les morts; d'autres en sont pris chez eux sans aucune cause connue" ${ }^{\text {. }}$ Épargné en 1832, le clergé perdit cette fois quatre prêtres, même si pour l'ensemble de la population le nombre des morts fut moins élevé. L'apport des prêtres se manifesta lors des épidémies aux niveaux des derniers sacrements, du réconfort spirituel, des invitations à la prudence.

\footnotetext{
${ }^{8}$ ACAM, 780-034, 832-3, 4 avril, D. Daby, secrétaire de la province du BasCanada.

${ }^{9} \mathrm{ACDL}$ (Archives du Centre diocésain à Longueuil), St-Constant, I, 834-2, 26 août, Jean-Olivier Chèvrefils à Lartigue.
} 
Quant aux soins hospitaliers en général, ils étaient dispensés surtout aux citadins par les quelques communautés féminines qui se vouaient alors à cette tâche. Des subventions gouvernementales étaient allouées annuellement, par exemple $3331 £$ en 1830 , spécialement en faveur des «fous, des enfants trouvés, des malades indigents, des immigrés» ${ }^{10}$. L'Hôtel-Dieu de Saint-Hyacinthe fut bâti en 1840, grâce à l'initiative du curé Edouard-Joseph Crevier et à la générosité des religieuses de l'Hôpital-Général de Montréal, dénommées localement «les Filles de la charitć". On a pu lire alors dans Le Canadien: "Honneur au clergé qui comprend si bien sa divine mission! Honneur au vénérable prêtre qui a conçu cette oeuvre et qui a pu vaincre tous les obstacles pour la mener à bien!» ${ }^{11}$

\section{VIS-À-VIS DES PAUVRES}

Les périodes marquées par les épreuves naturelles comportaient des effets néfastes sur les provisions financières des Canadiens. Un comité spécial du gouvernement releva, en janvier 1834, 2,243 familles réduites à la misère dans vingt-huit paroisses des comtés de Saguenay, Rimouski, Kamouraska, Bellechasse et de la Beauce. "Il n'est pas rare de voir des familles qui passent deux jours sans trouver la plus vile nourriture» ${ }^{12}$. En 1836, le curé de Carleton se plaint: "Les années ici vont de mal en pis... Il nous faut tuer plus de la moitié de nos animaux faute de fourrage. Les gelées ont empêché les patates, notre pain quotidien, et les blés de mûrir. Pour surcroît de malheur, des troupes innombrables de vermine rongent et mangent tout ce qui n'est pas engrangé»" ${ }^{13}$. Et ces disettes étaient partagées par les curés. «Un morceau de pain noir, quelques petits poissons, les mouches ou huîtres de l'endroit, crapauds ou poules de mer, et je ne sais quels autres insectes marins, voilà notre nourriture ${ }^{14}$, gémit le curé de Cacouna au printemps 1837.

Des habitants ne s'adressaient pas au gouvernement bas-canadien pour être aidés financièrement, sans que le curé ne se joignît à eux. Ce dernier convenait avec son évêque de la meilleure façon de procéder. Les rouages gouvernementaux ne fonctionnant pas rapidement et se

\footnotetext{
${ }^{10}$ La Minerve, 22 mars 1830.

${ }^{11}$ Le Canadien, 29 mai 1840.

${ }^{12}$ AAR (Archives de l'archevêché de Rimouski), Trois-Pistoles, 1834-1835, 6 févr. 1834, Louis 'Théophile Fortier, curé, à Plessis.

${ }^{13}$ AAR, Carleton, 1, 27 sept., Louis Stanislas Malo à Charles-Félix Cazeau, secrétaire de l'évêque de Québec.

${ }^{14}$ AAR, Cacouna, 1835-1849, 6 mai, Édouard Quertier, curé, à Cazeau.
} 
préoccupant bien plus d'empêcher les gens de quêter publiquement (loi de 1818) que d'enrayer le mal à la source, l'évêque et ses curés couraient au plus pressé.

Ils remédiaient de diverses façons à l'indigence des gens. Au lieu de vendre son blé, provenant des dîmes, au prix normal de $2 \$$ le minot, le curé François-Xavier Noiseux de Trois-Rivières le céda aux pauvres en 1805 à seulement $1 \$$. $\mathbf{M}^{\mathrm{gr}}$ Plessis fit distribuer environ $150 £$ aux pauvres de Saint-Eustache et de Saint-Benoît durant l'hiver 1813. Le curé Charles Berthelot procura 25 louis de ses biens à son évêque, afin que ce dernier les distribuât aux démunis: «Il faut dans la disette donner ce qu'on a épargné dans l'abondance. Quand d'autres crèvent de faim il n'est plus de saison de garder une poire pour la soif. Ceux qui ont épargné ont emmagasiné pour la Providence. Qu'ils démagasinent. La Providence leur viendra encore» ${ }^{15}$. En legs testamentaire, Louis Lamothe laissa 1000 livres françaises aux pauvres de la paroisse dont il était curé. Au préfet de la Propagande, le supérieur des Sulpiciens ne manqua pas de signaler en 1822 que la corporation du Séminaire soutenait les deux mille pauvres de Montréal.

Si les prêtres faisaient leur part en faveur des pauvres, que dire des fabriques? $\mathbf{M}^{\mathrm{gr}}$ Plessis suggéra à son évêque coadjuteur, qui de sa cure de Rivière-Ouelle supervisait les paroisses du bas Saint-Laurent, qu'en attendant l'aide éventuelle du gouvernement les marguilliers pourraient tirer la moitié de l'argent de leur coffre-fort pour l'achat de vivres en faveur des plus pauvres de leur paroisse. $\mathrm{M}^{\mathrm{gr}}$ Lartigue préférait pour sa part qu'une fabrique prêtât de l'argent plutôt que d'en donner pour l'achat des grains nécessaires à l'ensemencement; les plus riches pouvaient selon lui procurer aux plus pauvres ce dont ces derniers avaient besoin pour subsister. Par exemple, la fabrique de Saint-Hyacinthe consentit à prêter $250 £$ en 1836 aux familles pauvres pour ensemencer leur terre.

L'évêque de Montréal comme celui de Québec s'en tinrent finalement à ces orientations-ci: d'abord l'aide des riches par une collecte et par quelques initiatives particulières; puis des prêts par la fabrique pour l'ensemencement et la répartition de la quête de l'Enfant-Jésus en faveur des pauvres; enfin, peut-être un don de la fabrique, les trois derniers moyens nécessitant une résolution de fabrique. La Saint-Vincent de Paul, bien que déjà fondée à Paris en 1823, serait instaurée dans le Bas-

${ }^{15}$ ACDL, St-Luc, I, 813-2, 3 avril, à Plessis. 
Canada seulement le 12 novembre 1846 . Elle deviendrait le moyen privilégié grâce auquel les paroissiens de chaque milieu s'entraideraient financièrement.

Des projets sourdaient aussi d'individus bienveillants, sinon sous l'influence, du moins avec la participation d'un prêtre. En décembre 1808, une souscription fut envisagée à Québec, moyennant l'approbation des évêques catholique et anglican. L'argent recueilli servirait à l'achat de bois de chauffage pour les indigents des deux Églises, à raison des deux tiers pour les catholiques et d'un tiers pour les anglicans. En 1817, à Montréal, un comité spécial de dix hommes, incluant entre autres un pasteur de chacune des grandes Églises chrétiennes, fut constitué par une assemblée de personnes, préoccupées de prendre les mesures nécessaires pour soulager la misère de nombreux citoyens.

Dans un article de la Gazette de Québec, on avait lu en 1815 que la société avaient suffisamment évolué pour se rendre compte que le soutien des pauvres n'était pas seulement un "devoir religieux", mais aussi une "obligation légale». Et l'auteur ajoutait: «Les maisons d'industrie sont un excellent moyen d'enrayer le mal des pauvres" ${ }^{16}$. On se référait à un projet développé par «Les deux amis" les 18 février 1807 dans Le Courrier de Québec; ils y promouvaient l'existence de maisons de travail où les pauvres trouveraient de l'emploi et de maisons de correction où les oisifs et les vagabonds seraient forcés de travailler. Le plan comportait un ensemble de bâtiments où ces deux catégories de personnes seraient distinctes et subdivisées selon leur sexe. Ce projet à la fois philantropique et évangélique avait pour buts de remplir les devoirs d'aide humanitaire ou charitable, de rectifier les moeurs et d'enseigner aux infortunés les moyens de gagner honnêtement leur vie. L'établissement proposé consisterait en une école d'industrie.

L'idée fit son chemin. Une maison d'industrie fut fondée pour les pauvres de Montréal en 1819. La corporation s'engagea «à procurer de l'emploi à tous les pauvres, industrieux, qui n'en pourront trouver ailleurs et qui voudront se présenter pour en avoir" ${ }^{17}$; on retenait donc seulement la première catégorie des gens visés. Par ailleurs, à partir de ce jour, on appliqua le règlement municipal de ne plus mendier dans les rues. La générosité des citoyens, au lieu de s'exprimer auprès des mendiants, pourrait ainsi s'orienter vers cette nouvelle oeuvre sociale,

${ }^{16}$ La Gazette de Québec, 26 janv. 1815.

${ }^{17}$ L'Aurore, 29 mai. 
pour laquelle les biens légués par le fondateur, Conrad Marsteller, ne suffisaient pas. Une collecte domiciliaire eut lieu du ler au 19 juillet et le système de collecteurs de quartier subsisterait fort longtemps, sans compter les quêtes faites dans les églises de la ville. Au début d'octobre, la nouvelle institution avait porté les fruits escomptés. Les vagabonds étaient disparus des rues, alors que les vrais pauvres subvenaient à leurs besoins par le travail. Beaucoup plus tard, le 16 décembre 1836, on compterait 181 personnes admises pour l'hiver à la maison d'industrie: 85 Canadiens, 72 Irlandais, 12 Anglais, 9 Écossais, 2 Italiens ou, d'un autre angle: 39 femmes, 51 hommes, 44 garçons, 30 filles, 14 nourrissons. En février 1838, 900 personnes recevaient un secours journalier de la part du comité responsable de la maison d'industrie, à laquelle s'était joint un service de dépannage.

Cette oeuvre suscita d'autres initiatives semblables. En décembre 1828, les Sulpiciens convoquèrent des citoyens et ils déterminèrent ensemble les moyens de secourir les pauvres pendant les mois suivants. Il fut décidé qu'une souscription serait ouverte et que le produit servirait à payer les pauvres qui casseraient la pierre offerte gratuitement par les magistrats pour macadamiser les rues de Montréal. La pierre cassée serait achetée par les dirigeants de la ville et l'argent ainsi obtenu serait utilisé l'année suivante pour salarier les pauvres qui s'adonneraient à un travail similaire. On s'aperçut cependant que ces indigents manquaient de vêtements pour travailler au froid. Un porte à porte fut alors organisé et l'on remédia à ce problème.

À l'approche de 1830 surgirent à divers endroits des associations de Dames de la charité. À Montréal, elles tinrent leur première assemblée en décembre 1827 sous la présidence de la baronne de Longueuil et avec l'appui des Sulpiciens. Leur objectif consista à secourir les malheureux pendant les rigueurs de l'hiver. Dons, souscriptions, collectes apparurent comme les premiers moyens de mener à bien cette initiative évangélique; dès l'année suivante, les bazars deviendraient une source importante de revenus. L'exemple de Montréal fut suivi à Québec, en particulier dans la paroisse Saint-Roch, à Saint-Jean en 1832, à La Malbaie et à Rimouski en 1837. On souhaitait voir s'instaurer partout une association des Dames de la charité. "L'on y verrait disparaître en peu de temps l'indigence et les conséquences funestes dont elle n'est malheureusement que trop souvent la cause et la source» ${ }^{18}$.

\footnotetext{
18 La Minerve, 19 nov. 1832.
} 
La persévérance de l'une des associées de Montréal, Émilie Tavernier, connue sous le nom de veuve Jean-Baptiste Gamelin, auprès des femmes âgées ou infirmes (elle en accueillit vingt-huit en 1837 et trente-deux en 1840), la réapparition de l'Oeuvre de la soupe à Montréal, qui rendrait service à neuf cents pauvres en 1838, le succès financier $(450 f)$ du bazar de 1837 dans la paroisse Saint-Roch à Québec au profit des douze cents pauvres dont s'occupait le curé David Têtu ressortent comme des signes d'une grande pratique de la charité chrétienne envers les pauvres. L'apport des femmes y étaient prédominant.

\section{EN FAVEUR DES MARGINAUX}

\section{Les délaissés}

À Québec, les Dames de la charité s'occupèrent dès 1828 de trouver un refuge aux orphelins. En 1832, elles quêtèrent tous les effets et articles pouvant servir à ces jeunes. À Montréal, les dames s'ajustèrent elles aussi aux besoins; elles établirent en 1832 un hospice pour les orphelins du choléra; il s'en trouvait déjà quarante le 29 octobre. Ils y étaient instruits et finalement placés chez d'honnêtes gens. On se préoccupait aussi des 172 veuves et de leurs 520 orphelins qui demeuraient à domicile et qui auraient besoin de vêtements et de nourriture durant l'hiver 1832-1833.

Le placement des orphelins dans des familles d'adoption s'effectuait avec respect et attention. Le curé Baillargeon de Québec n'envoya pas six orphelins à la Rivière-Ouelle, sans que le curé de l'endroit ne lui transmît préalablement le nom de chaque famille d'accueil et le désir de chacune d'avoir un garçon ou une fille. Une pièce d'identité accompagnait chacun des jeunes, afin que l'on n'oubliât pas son origine.

Les Dames de la charité de Québec en arrivèrent à sectionner leurs oeuvres. L'une s'appela la Société charitable pour le soulagement des orphelins. Le 30 juin 1834 , la résidence transitoire de ces derniers en comptait vingt-quatre. Un local fut acheté le 5 novembre suivant; il servirait aussi d'école pour les petites filles indigentes.

Compte tenu de l'analphabétisme fort répandu $(78,000$ signatures étaient des croix sur les 87,000 qui étaient apposées au bas de la requête contre le régime Dalhousie en 1827), des Montréalais projettent en 1831 de constituer avec la collaboration de l'ecclésiastique Amable Daniel Duchaîne et sous les auspices de $\mathbf{M}^{\text {gr }}$ Lartigue «une institution 
qui aura pour objet de donner aux ouvriers canadiens une instruction qui puisse leur donner plus d'avantages dans l'exercice de leurs métiers et dans la conduite de leurs affaires" ${ }^{19}$. L'évêque prêta des locaux de sa nouvelle maison pour que s'y établitt une école d'arts et métiers. La compétence de Duchaîne, âgé de 58 ans, était reconnue par le gouvernement, du moins pour la construction des ponts. Bien qu'il ne fût jamais ordonné prêtre, il enseigna gratuitement aux jeunes gens en arts mécaniques et il en aida d'autres "à exercer leurs arts et métiers respectifs avec plus d'avantages pour eux-mêmes et pour la société» ${ }^{20}$.

Parmi les analphabètes se trouvaient les sourds-muets. Leur initiation aux sacrements requérait déjà une attention particulière de la part des prêtres. La grammaire de l'abbé Sicard, dont le seul exemplaire circulait entre l'évêché et telle ou telle paroisse, servait d'instrument d'évangélisation en même temps que d'éducation de ces personnes handicapées. Une institution s'ouvrit à Québec le 15 juin 1831 sous la direction d'un certain Macdonald, grâce à la générosité financière de divers bienfaiteurs. Le gouvernement se dit prêt à s'en occuper, une fois qu'un recensement aurait été réalisé. On compta 393 sourds-muets dans le Bas-Canada à la fin de 1831. Alors que l'initiative de Québec avait finalement suscité l'ouverture d'une résidence gouvernementale, une autre surgit du Séminaire de Saint-Hyacinthe. Les sourds-muets y furent accueillis en 1836 dans une classe spéciale, aux mêmes conditions que les autres élèves. Le Canadien soutint que ce service coûterait ainsi beaucoup moins cher au gouvernement, s'il voulait y contribuer, que sa propre institution, et que ce serait beaucoup plus efficace que les tentatives antérieures.

Dans toute société, les prostituées constituent un groupe marginal particulier. Huguette Latour-McDonnell, pieuse, respectable et industrieuse, instaura à Montréal en 1829, avec l'avis et l'appui de $\mathbf{M}^{\mathrm{gr}}$ Lartigue, une maison pour «les femmes ou filles publiquement débauchées, qui veulent se convertir». Un an plus tard, elle abritait "plus de trente de ces créatures, qui se conduisent bien, gagnent leur vie en travaillant»" ${ }^{21}$. Les Sulpiciens y assuraient le ministère pastoral. Une loi d'incorporation de cet Institut des filles repenties fut voté en mai 1833 et sanctionné à Londres l'année suivante. À l'occasion d'une visite à Québec, la directrice reçut entre autres dons pour son oeuvre la somme de $£ 20$ des mains de $\mathbf{M}^{\mathrm{gr}}$ Signay.

${ }^{19}$ ACAM, 901.026, 831-1, 29 nov., Duchaîne à Lartigue.

${ }^{20} \mathrm{Ibid}$., 832-2, 15 janv., Avis public.

${ }^{21}$ ACAM, RLL (Registres de lettres de $\mathrm{M}^{\mathrm{gr}}$ Lartigue), 5, 352, 27 oct. 1830, à Panet. 
Au printemps 1836, il s'y trouvait cinquante-deux filles, certaines y étant depuis quelques années. Trois cents femmes y avaient séjourné depuis sept ans.

Un bien petit nombre, écrivait La Minerve à ce sujet, en sont sorties pour retourner au vice... beaucoup sont retournées dans leur famille, dont elles ont fait l'exemple et qui sont maintenant des femmes vertueuses... Souvent une malheureuse jeune fille est jetée dans le vice par la misère, par une faiblesse dont elle ne calcule pas les conséquences... La société entière semble les rejeter.

Un asile était indispensable; or il existait. "Nous appelons de nouveau le public, poursuivait $\mathrm{La}$ Minerve, à seconder" Mme McDonnell «dans ses efforts, jusqu'à ce que l'établissement ait quelques fonds avec lesquels il puisse se soutenir à l'aide du travail des filles»" ${ }^{22}$. Les hommages fusaient de toutes parts en faveur de cette oeuvre. Un journaliste de L'Ami du peuple, de l'ordre et des lois, invité à visiter l'institution, révisa tous ses préjugés sur les organismes de charité et en arriva à écrire ce qui suit :

Il existe des personnes... qui dans le silence et sans ostentation portent du secours aux maisons infortunées, où se trouve le malade sans secours, l'orphelin abandonné et la pauvre veuve souffrant les douleurs de la faim... J'ai cru pendant quelque temps que les protestants étaient les vrais dispensateurs de la Charité et que les catholiques ne la faisaient qu'en jetant des sols aux vagabonds nombreux qui parcourent nos rues. ${ }^{23}$

L'oeuvre si bien cotée ne disparut pas moins le 22 juin 1837, faute d'argent. Elle avait rendu de grands services.

Et qu'en était-il des prisonniers? Les journaux rapportent surtout les punitions publiques, en particulier les pendaisons. Le Courrier de Québec décrit ainsi les derniers moments des prisonniers exécutés.

Ils pleurent, crient et prient Dieu toute la journée... Ils communient avant d'aller à l'échafaud et s'y avancent la corde au cou, un bonnet sur la tête et un bouquet au côté, exhortés par des ministres de leur religion, même par des prêtres catholiques, et chantant des hymnes. ${ }^{24}$

${ }^{22}$ La Minerve, 2 mai 1836.

${ }^{23}$ L'Ami du peuple, de l'ordre et des lois, 7 mai 1836.

${ }^{24}$ Le Courrier de Québec, 24 août 1818. 
La Gazette des Trois-Rivières relève l'exemple d'un jeune, mort «avec beaucoup de pénitence et de résignation. On ne saurait trop admirer la constance et la charité du digne prêtre qui depuis six semaines s'est occupé sans relâche à le préparer à sa triste fin»" ${ }^{25}$.

Charles Alarie et Thomas Thomas, assistés par des prêtres de la cure de Québec, "moururent repentants, sollicitant les prières du concours nombreux assemblé à cette occasion et les exhortant à éviter les voies qui les avaient amenés à une fin aussi prématurée" ${ }^{26}$, à cause du vol d'un bateau dans le port. Un motif fréquent d'exécution était le vol, surtout quand il s'agissait d'un vol sacrilège.

Vers 1830 se constitua à Québec une institution charitable qui promouvait "l'éducation et l'industrie parmi les personnes renfermées dans les prisons" et leur inculquant "les préceptes de la morale et de la religion"; cela produisit «de grands biens" ${ }^{27}$. À Montréal, $\mathbf{M}^{\text {gr }}$ Lartigue insista auprès du supérieur des Sulpiciens pour qu'un prêtre célébrât la messe dominicale à la prison. À la suite des troubles de 1837 et de $1838, M^{g r}$ Bourget se soucia de visiter lui-même les prisonniers, tout en ayant à coeur de soulager la misère des pauvres familles, privées d'un époux et d'un père. $\mathbf{M}^{\mathrm{gr}}$ Lartigue invita en vain les dirigeants gouvernementaux à libérer les prisonniers politiques. Une fois plusieurs d'entre eux exilés aux Bermudes et en Australie, il intervint efficacement auprès du vicaire apostolique de Sydney pour qu'il s'occupât de ceux qui étaient expatriés en ce dernier pays. $\mathbf{M}^{\mathrm{gr}}$ Bourget insista pour sa part auprès de Londres en faveur du retour de tous les exilés, qui s'effectua de fait peu après.

L'évêque auxiliaire de Montréal tira profit de son ministère carcéral pour indiquer au gouverneur Colborne des points de réforme à la prison de Montréal: classer soigneusement les incarcérés; empêcher la communication entre les jeunes et les vieux, entre les grands criminels et les fautifs occasionnels; assurer une cellule séparée et fermée à chaque prisonnier, du moins la nuit; faire travailler les prisonniers selon leur capacité; empêcher les prostituées d'aller et venir dans les prisons.

$\mathrm{M}^{\mathrm{gr}}$ Bourget s'intéressa aussi aux aliénés mentaux. "Il est bien honteux et scandaleux pour une ville riche comme Montréal de n'avoir pas un asile pour des êtres si malheureux*. En attendant que la Législa-

${ }^{25}$ La Gazette des Trois-Rivières, 3 nov. 1818.

${ }^{26}$ La Gazette de Québec, 3 nov. 1818.

${ }^{27}$ L'Écho du pays, 7 janv. 1836. 
ture y pourvût, il demanda à la supérieure de l'Hôpital-Général de leur aménager des appartements, d'autant plus que «la religion est faite pour subvenir à toutes les misères de la pauvre humanité" ${ }^{28}$. Cette nouvelle oeuvre ne tarderait pas à produire ses fruits.

\section{Les migrants}

L'immigration vint surtout de la Grande-Bretagne. Déjà en 1818 , des catholiques étrangers arrivaient par milliers, en particulier de l'Irlande. Le comité des émigrants de Montréal requit la collaboration des pasteurs de chaque dénomination religieuse, pour procurer du travail à des domestiques des deux sexes, des journaliers et autres travailleurs immigrants. À l'automne $1820, \mathrm{M}^{\mathrm{gr}}$ Plessis invita tous ses curés par une lettre circulaire à placer au moins une famille irlandaise dans leur paroisse. "Ces pauvres gens périssent de froid et de misère dans les rues" ${ }^{29}$. L'accueil fut réticent, car des paroissiens d'un peu partout ainsi que des curés discernaient plus d'inconvénients que d'avantages à l'arrivée d'étrangers dans leur milieu.

La décennie de 1830 connut une recrudescence d'immigration irlandaise. De 1829 à 1835 inclusivement, entrèrent dans le Bas-Canada 128,578 Irlandais sur un total d'immigrants britanniques de 211,159 . Ils s'établirent plutôt dans les cantons et dans les villes, où s'effectuaient de grandes entreprises publiques, tel le creusage de canaux. Ces immigrants, trompés dans leur pays d'origine sur ce qui les attendait en Amérique, se trouvaient généralement dans des conditions inhumaines de travail et de logement. Le clergé se préoccupa de leur assurer des célébrations dominicales propres à eux, à Québec, Trois-Rivières, Chambly, Beauharnois. Les évêques favorisèrent aussi le regroupement des Irlandais, en vue de rendre possibles et viables leurs liens fraternels et communautaires. La Société des émigrants, qui se constitua de façon oecuménique en juillet 1831 à Montréal, compta parmi ses membres des représentants de $\mathbf{M}^{\mathrm{gr}}$ Lartigue. Son double objectif consistait à soulager les immigrés en détresse et à les envoyer dans différentes parties de la province. Il s'agissait là d'une oeuvre plus nécessaire et mieux organisée qu'ailleurs, quoique généralisée dans l'ensemble de la province.

\footnotetext{
${ }^{28} \mathrm{ACAM}, R L B, 1,125,20$ déc. 1837, à Rév. Mère Beaubien.

${ }^{29}$ Desbras, Les Irlandais dans Sainte-Anne-du-Sud, dans BRH, 28, 10 (oct. 1922) p. 289.
} 
Par contre, des habitants d'origine canadienne quittaient leur pays. Déjà vers 1808-1810, des curés entretenaient leurs évêques sur les malheureux effets du travail saisonnier de nombreux jeunes gens aux ÉtatsUnis. Le phénomène d'émigration débuta surtout en 1837 et en 1838 , compte tenu de la crise agricole, commerciale et politique qui sévissait alors dans le Bas-Canada. L'arrivée des immigrants britanniques n'y était pas étrangère. $\mathrm{M}^{\mathrm{gr}}$ Bourget saisit tout de suite la gravité du problème.

Dès l'été 1839, l'évêque de Montréal recensa avec l'aide de ses curés le nombre de leurs paroissiens francophones partis vers le pays voisin depuis l'automne précédent. Il apparut que le phénomène n'était pas encore grave et qu'il se limitait en bonne partie à des hommes qui s'y rendaient au printemps pour travailler et qui en revenaient à l'automne. Mais l'objectif de l'opération était tronqué, car le gouverneur Colborne cherchait à savoir par ce biais combien de Canadiens se trouvaient outre frontières parce qu'ils avaient participé aux troubles ou qu'ils envisageaient de se soulever de nouveau. De la paroisse d'Iberville, l'une des plus marquées par l'émigration, le curé répondit que cent soixante-quatre individus étaient rendus aux États-Unis depuis l'automne 1838, dont trente-quatre familles. Les uns y étaient allés pour gagner leur vie, d'autres s'étaient éloignés de diverses vexations, enfin certains craignaient des châtiments bien mérités de la part du gouvernement. Seules trois ou quatre familles se proposaient cependant de ne plus revenir.

La réponse de Pierre-Marie Mignault, curé de Chambly, fut plus explicite que celle des autres curés, étant donné son expérience de missions qu'il accomplissait annuellement auprès des émigrés canadiens. " $\mathrm{La}$ cause de cette émigration serait le haut prix qu' on offre». On pourrait prévenir cette saignée en allouant aux Canadiens des terres dans les cantons et de quoi subsister deux ou trois ans. Il faudrait aussi y construire des chemins qui rejoindraient les grandes routes. Selon lui, plusieurs milliers de Canadiens avaient déjà émigré du Bas-Canada, en particulier vers Chicago. Pour la plupart, il s'agissait de journaliers, mais on y comptait aussi des cultivateurs et des menuisiers. «Les moeurs s'y perdent, la santé n'y gagne pas et les Canadiens qui reviennent sont souvent plus pauvres», malgré leur «air d'indépendance, de suffisance et d'orgueil qui leur sied assez mal» ${ }^{30}$.

${ }^{30} \mathrm{ACDL}$, St-Joseph de Chambly I, 849-1, 5 mars, à Bourget. 
Le phénomène de la migration prendrait de l'ampleur; le clergé continuerait de suppléer tant bien que mal aux lacunes et aux méfaits des politiques gouvernementales.

\section{Les alcooliques}

Les Sociétés de tempérance, instaurées aux États-Unis en 1827 , se propagèrent en Angleterre l'année suivante et s'élevaient déjà à une centaine dans le Haut-Canada en 1831. Cette année-là, une association se fonda à Québec, composée surtout de membres anglophones, comme cela avait été le cas à Montréal trois ans plus tôt. Le Canada Temperance Advocate parut à partir du ler mai 1835, sans lien avec une Église ou un parti politique. Son éditeur avait tout de même demandé l'appui de $\mathrm{M}^{\mathrm{gr}}$ Lartigue. À Sherbrooke, en 1837, se constitua la Irish Philantropic Society, dont les membres s'empêchaient de boire toute liqueur spiritueuse.

Charles Chiniquy, nommé curé à Beauport en 1838, constata que la plupart des habitants étaient réduits à la pauvreté à cause des boissons alcooliques. Il affirma qu'une valeur de $40000 £$ avaient été perdue en propriétés par les paroissiens à cause de leur intempérance. Il y fonda une Société de tempérance le 29 mars 1840. L'année suivante, les sept auberges avaient fait place à sept écoles et douze cents personnes faisaient leurs pâques au lieu des trois cents de 1838. Chiniquy fut aussi l'auteur d'une carte de tempérance imprimée, sur laquelle étaient écrits le nom de l'associé, la date de son engagement à l'abstinence et la signature du çuré. D'autres prêtres prêchèrent la tempérance avec succès: Pierre Beaumont, Michel Duchesne, David-Henri Têtu, Alexis Mailloux et Édouard Quertier, de qui est venue l'idée de la croix noire.

La formule d'engagement, propagée par Chiniquy, consistait en celle-ci:

Je m'engage solennellement et publiquement d'éviter l'intempérance et de ne jamais fréquenter les cabarets. Je ne ferai jamais usage de boissons fortes sans une absolue nécessité; et si pour devenir tempérant il me faut renoncer à toute espèce de boissons, je m'y engage; je promets aussi de faire tout en mon pouvoir par mes paroles et par mes exemples, pour que mes parents et mes amis en fassent autant. ${ }^{31}$

${ }^{31}$ Trudel, Marcel, Chiniquy, Trois-Rivières, Éditions du Bien Public, 1955, p. 32. 
En 1841, le curé va plus loin et propose aux plus courageux de s'abstenir même de la bière et du vin, ce que signèrent huit cent douze des treize cents paroissiens, ayant l'âge de raison et déjà engagés dans la tempérance.

Les sociétés incluaient deux ordres: celui de l'abstinence complète et celui de l'abstinence partielle. Le président de chacune des sociétés devint de plus en plus souvent le curé de la paroisse. Le clergé s'était mis à la tête de cet élan populaire, ce qui en garantissait le succès selon un rédacteur de L'Aurore des Canadas. L'expansion du mouvement fut favorisé par la prédication de $\mathbf{M}^{\text {gr }}$ de Forbin-Janson. Les cartes de tempérance arboraient en exergue les fruits de la tempérance: «bonheur domestique, santé de l'âme et du corps, félicité éternelle» et ceux de l'intempérance: "ruine des familles, pauvreté, infirmité, crime, folie, mort prématurée, mort éternelle" ${ }^{32}$. L'amélioration générale de la société s'en ressentit grandement.

\section{CONCLUSION}

Alors que le clergé cherchait à rendre service à de nombreuses personnes défavorisées et qu'il le faisait par souci évangélique, la situatịon sociale du Bas-Canada se transformait beaucoup. Des conflits ouvriers, tel celui du canal de Lachine, se déclarèrent peu après 1840 . Les patrons et les ouvriers se tiendraient tête de plus en plus fréquemment. Mais les misères humaines subsisteraient et la société tarderait à y remédier. L'Église, sans cesse confrontée avec des personnes marquées par ces problèmes, demeure accueillante à tous les déshérités. Le clergé et les communautés religieuses continueraient d'instaurer les institutions jugées nécessaires pour aider ces derniers. Il en ressortirait une pastorale caritative mieux organisée. Les dirigeants politiques ne se presseraient d'ailleurs pas de préciser et de réaliser ce qui relevait d'eux dans le domaine de la promotion humaine.

Lucien Lemieux, prêtre

Diocèse de Saint-Jean-Longueuil

Saint-Hubert

${ }^{32}$ ASQ, Lettres-Carton $W, 30,22$ déc. 1840, description d'une carte de tempérance. 